\title{
Analogue of the Goldberg-Sachs Theorem
}

\section{GARRY LUDWIG}

Communicated by T. Y. Thomas

Introduction. Recently various existence theorems [1, 2, 3] have been found for a tensor $H$ having the algebraic properties of a vacuum Riemann tensor and satisfying

$$
g^{\rho \sigma} H_{\alpha \rho \beta \sigma}=g^{\rho \sigma} H_{\alpha \rho \beta \sigma}^{*}=0
$$

and

$$
\nabla^{\rho} H_{\rho \sigma \lambda \mu}^{*}=0,
$$

where $H^{*}$ is the dual of $H$ and $\nabla$ is the covariant derivative. The Weyl tensor corresponding to a space-time with metric $g_{\rho \sigma}$ will be denoted by $W$.

We shall prove the following:

Theorem. Given a congruence of geodesic and shearfree null curves there exists a tensor $H$ with the properties described above which is, in addition, algebraically general, if and only if the Weyl tensor vanishes.

An analogue of the Goldberg-Sachs Theorem [4] then follows as a

Corollary. In a space-time with non-vanishing Weyl tensor $W$ a tensor $H$ with the properties described above is algebraically special if and only if there exists a congruence of geodesic and shearfree null curves.

Newman-Penrose method. The Newman-Penrose [5] method will be used to prove these assertions. At each point in space-time we choose a null-tetrad $k_{\alpha}, t_{\alpha}, t_{\alpha}, m_{\alpha}$ (obeying the normalization conditions $k_{\alpha} m^{\alpha}=1, t_{\alpha} t^{\alpha}=-1$ ) as basis for the tangent space of vectors. This tetrad corresponds to a basis $k_{A}, m_{A}$ (with $k_{A} m^{A}=1$ ) in the tangent spin-space. The Weyl tensor $W$ is characterized by a completely symmetric spinor [6] $\Psi_{A B C D}$ wihch can be regarded as an element of a five dimensional complex linear space [7] $E_{5}$. The spin-space basis induces a basis in $E_{5}$ in which $\Psi_{A B C D}$ can be written as

$$
\begin{gathered}
\Psi_{A B C D}=\Psi_{0} m_{A} m_{B} m_{C} m_{D}-4 \Psi_{1} k_{A} m_{B} m_{C} m_{D}+6 \Psi_{2} k_{A} k_{B} m_{C} m_{D} \\
-4 \Psi_{3} k_{A} k_{B} k_{C} m_{D}+\Psi_{4} k_{A} k_{B} k_{C} k_{D} . \\
185
\end{gathered}
$$

Indiana University Mathematics Journal, Vol. 20, No. 2 (1970). 
The coefficients are the dyad components of the Weyl tensor, introduced by Newman and Penrose.

A change of spin-frame

$$
\begin{aligned}
k_{A}^{\prime} & =a k_{A}+b m_{A}, \quad(a d-b c=1) \\
m_{A}^{\prime} & =c k_{A}+d m_{A}, \quad(\quad)
\end{aligned}
$$

(or, equivalently, a change of null tetrad in space-time) gives rise to a change of basis [7] in $E_{5}$. We shall need the transformation laws for the dyad components of an element of $E_{5}$ under certain kinds of spin-frame transformations. We have for a null rotation about $k^{\alpha}(a=d=1, b=0)$

(3)

$$
\begin{aligned}
& \Psi_{0}^{\prime}=\Psi_{0}, \\
& \Psi_{1}^{\prime}=c \Psi_{0}+\Psi_{1}, \\
& \Psi_{2}^{\prime}=c^{2} \Psi_{0}+2 c \Psi_{1}+\Psi_{2}, \\
& \Psi_{3}^{\prime}=c^{3} \Psi_{0}+3 c^{2} \Psi_{1}+3 c \Psi_{2}+\Psi_{3}, \\
& \Psi_{4}^{\prime}=c^{4} \Psi_{0}+4 c^{3} \Psi_{1}+6 c^{2} \Psi_{2}+4 c \Psi_{3}+\Psi_{4},
\end{aligned}
$$

for a null rotation about $m^{\alpha}(a=d=1, c=0)$

$$
\begin{aligned}
& \Psi_{0}^{\prime}=b^{4} \Psi_{4}+4 b^{3} \Psi_{3}+6 b^{2} \Psi_{2}+4 b \Psi_{1}+\Psi_{0}, \\
& \Psi_{1}^{\prime}=b^{3} \Psi_{4}+3 b^{2} \Psi_{3}+3 b \Psi_{2}+\Psi_{1}, \\
& \Psi_{2}^{\prime}=b^{2} \Psi_{4}+2 b \Psi_{3}+\Psi_{2}, \\
& \Psi_{3}^{\prime}=b \Psi_{4}+\Psi_{3}, \\
& \Psi_{4}^{\prime}=\Psi_{4},
\end{aligned}
$$

and for $d=1 / a, b=c=0$

$$
\begin{aligned}
& \Psi_{0}^{\prime}=a^{4} \Psi_{0}, \\
& \Psi_{1}^{\prime}=a^{2} \Psi_{1}, \\
& \Psi_{2}^{\prime}=\Psi_{2}, \\
& \Psi_{3}^{\prime}=1 / a^{2} \Psi_{3}, \\
& \Psi_{4}^{\prime}=1 / a^{4} \Psi_{4} .
\end{aligned}
$$

To describe the variation of spinors and tensors with points in space-time directional derivatives along the null-tetrad vectors are introduced by

$$
\begin{aligned}
D & =k^{D} \bar{k}^{i} \nabla_{D i}=k^{\alpha} \nabla_{\alpha}, \\
\delta & =k^{D} \bar{m}^{i} \nabla_{D i}=t^{\alpha} \nabla_{\alpha}, \\
\bar{\delta} & =\bar{k}^{i} m^{D} \nabla_{D i}=\bar{t}^{\alpha} \nabla_{\alpha}, \\
\Delta & =\bar{m}^{i} m^{D} \nabla_{D i}=m^{\alpha} \nabla_{\alpha},
\end{aligned}
$$


where $\nabla_{D i}$ is the spinor operator corresponding to $\nabla_{\alpha}$. Since the directional derivatives of $k_{A}$ and $m_{A}$ are again elements of spin space they can be expressed in terms of the basis spinors:

$$
\begin{aligned}
D k_{A} & =\epsilon k_{A}-\kappa m_{A}, \\
\delta k_{A} & =\beta k_{A}-\sigma m_{A}, \\
\bar{\delta} k_{A} & =\alpha k_{A}-\rho m_{A}, \\
\Delta k_{A} & =\gamma k_{A}-\tau m_{A}, \\
D m_{A} & =\pi k_{A}-\epsilon m_{A}, \\
\delta m_{A} & =\mu k_{A}-\beta m_{A}, \\
\bar{\delta} m_{A} & =\lambda k_{A}-\alpha m_{A}, \\
\Delta m_{A} & =\nu k_{A}-\gamma m_{A} .
\end{aligned}
$$

The components are called spin coefficients. There are only twelve independent ones due to identities of the form $D\left(k_{A} m^{A}\right)=0$. There are eighteen different compatibility conditions on equations (6). To obtain them one must first work out the commutators of the basis spinors with the help of the Ricci identity. The eighteen "Ricci identities" are equations (4.2) in [5]. They are too long to be repeated here.

The compatibility conditions on these equations, in turn, yield the component equations of the Bianchi identity. In empty space they are the eight components of

$$
\nabla^{\rho} W_{\rho \sigma \lambda \mu}^{*}=0
$$

namely equations (4.5) in [5].

Since these equations are quite complicated it is gratifying to have some checks on their accuracy. The fact that equations (4.5) in [5] can be obtained independently both as components of (7) and as compatibility conditions on the Ricci identities (in the latter case each equation is obtained several times) gives the best check of all. The computations, however, are extremely long. A second check that can be applied not only to the "Bianchi identities" but also to the Ricci identities, the commutator relations, and the compatibility conditions (see below) is the following. A transformation of spin-frame with $a=d=0, b=c=i$ gives rise to the transformations

$$
\begin{gathered}
D^{\prime}=\Delta, \quad \delta^{\prime}=\bar{\delta}, \quad \bar{\delta}^{\prime}=\delta, \quad \Delta^{\prime}=D, \quad \Psi_{0}^{\prime}=\Psi_{4}, \quad \Psi_{1}^{\prime}=\Psi_{3}, \quad \Psi_{2}^{\prime}=\Psi_{2}, \\
\Psi_{3}^{\prime}=\Psi_{1}, \quad \Psi_{4}^{\prime}=\Psi_{0}, \quad \kappa^{\prime}=-\nu, \quad \sigma^{\prime}=-\lambda, \quad \rho^{\prime}=-\mu, \quad \tau^{\prime}=-\pi, \\
\epsilon^{\prime}=-\gamma, \quad \beta^{\prime}=-\alpha, \quad \alpha^{\prime}=-\beta, \quad \gamma^{\prime}=-\epsilon, \quad \pi^{\prime}=-\tau, \quad \mu^{\prime}=-\rho, \\
\lambda^{\prime}=-\sigma, \quad \nu^{\prime}=-\kappa, \quad k_{\alpha}^{\prime}=m_{\alpha}, \quad m_{\alpha}^{\prime}=k_{\alpha}, \quad t_{\alpha}^{\prime}=\bar{t}_{\alpha}, \quad \bar{t}_{\alpha}^{\prime}=t_{\alpha} .
\end{gathered}
$$

Equation (7) is unaffected by this transformation, but not so its component 
equations. For example, the first of equations (4.5) in [5] transforms into the last.

Comparison of equations (2) and (7) shows that the component equations of (2) are obtained from (4.5) in [5] by substituting $\Phi$ 's for $\Psi$ 's, where $\Phi_{\boldsymbol{A} B C D}$ is the element of $E_{5}$ corresponding to $H$ :

$$
\begin{aligned}
& D \Phi_{1}-\bar{\delta} \Phi_{0}+3 \kappa \Phi_{2}-(2 \epsilon+4 \rho) \Phi_{1}+(4 \alpha-\pi) \Phi_{0}=0 \\
& \delta \Phi_{1}-\Delta \Phi_{0}+3 \sigma \Phi_{2}-(4 \tau+2 \beta) \Phi_{1}+(4 \gamma-\mu) \Phi_{0}=0 \\
& D \Phi_{2}-\bar{\delta} \Phi_{1}+2 \kappa \Phi_{3}-3 \rho \Phi_{2}+(2 \alpha-2 \pi) \Phi_{1}+\lambda \Phi_{0}=0 \\
& \delta \Phi_{2}-\Delta \Phi_{1}+2 \sigma \Phi_{3}-3 \tau \Phi_{2}+(2 \gamma-2 \mu) \Phi_{1}+\nu \Phi_{0}=0 \\
& D \Phi_{3}-\bar{\delta} \Phi_{2}+\kappa \Phi_{4}+(2 \epsilon-2 \rho) \Phi_{3}-3 \pi \Phi_{2}+2 \lambda \Phi_{1}=0 \\
& \delta \Phi_{3}-\Delta \Phi_{2}+\sigma \Phi_{4}+(2 \beta-2 \tau) \Phi_{3}-3 \mu \Phi_{2}+2 \nu \Phi_{1}=0 \\
& D \Phi_{4}-\delta \Phi_{3}+(4 \epsilon-\rho) \Phi_{4}-(4 \pi+2 \alpha) \Phi_{3}+3 \lambda \Phi_{2}=0 \\
& \delta \Phi_{4}-\Delta \phi_{3}+(4 \beta-\tau) \Phi_{4}-(4 \mu+2 \gamma) \Phi_{3}+3 \nu \Phi_{2}=0
\end{aligned}
$$

It is the compatibility conditions on these equations that will help prove our theorem.

Compatibility conditions. The compatibility conditions on equation (8) that we need here are

$$
\begin{gathered}
\left(\Phi_{0} \Psi_{2}-\Phi_{2} \Psi_{0}\right)+k^{A} k^{B} k^{C} k^{D}\left[5 \Phi_{(A B \mid E F} \Psi_{C D)}^{E F \mid}+S_{A B C D}\right]=0, \\
\Phi_{0} \Psi_{3}-3 \Phi_{1} \Psi_{2}+3 \Phi_{2} \Psi_{1}-\Phi_{3} \Psi_{0}=0 \\
\Phi_{0} \Psi_{4}-2 \Phi_{1} \Psi_{3}+2 \Phi_{3} \Psi_{1}-\Phi_{4} \Psi_{0}=0 \\
\Phi_{1} \Psi_{4}-3 \Phi_{2} \Psi_{3}+3 \Phi_{3} \Psi_{2}-\Phi_{4} \Psi_{1}=0 \\
\left(\Phi_{4} \Psi_{2}-\Phi_{2} \Psi_{4}\right)+m^{A} m^{B} m^{C} m^{D}\left[5 \Phi_{(A B \mid E F} \Psi_{C D)}^{E F \mid}+S_{A B C D}\right]=0
\end{gathered}
$$

where

$S_{A B C D}=[\bar{\delta} \delta-\Delta D+(\gamma+\bar{\gamma}-\bar{\mu}) D+(\bar{\beta}-\bar{\tau}-\alpha) \delta-\tau \bar{\delta}+\rho \Delta] \Phi_{A B C D}$.

These equations are, in fact, the components in $E_{5}$ of

$$
\Phi_{A B C D}-6 \Phi_{(A B}^{\mid E F} \Psi_{E F \mid C D)}=0 .
$$

Equation (9), for example, is obtained by letting $\delta$ operate on the first of equations (8), $D$ on the second, and subtracting the results. Then follows a long and tedious calculation using the commutator relations (4.4) in [5] and the Ricci identities (4.2) in [5] over and over again.

The basis tetrad can always be chosen in such a way that $\Phi_{0}=0$. If this is done the only compatibility conditions on the Bianchi identities that need to be considered are (9)-(12) (with $\Phi_{0}=0$ ). 
The Goldberg-Sachs Theorem states that in a vacuum the Weyl tensor $W$ is algebraically special if and only if there exists a shearfree null geodesic congruence. The proof depends heavily on equation (9). If $W$ is algebraically special the null tetrad can be chosen in such a way that $k^{\alpha}$ is the repeated DebeverPenrose vector of $W$. It follows immediately from (8) that $\kappa=\sigma=0$, i.e. that $k^{\alpha}$ is geodesic and shearfree. Conversely, if there exists a geodesic and shearfree congruence of null vectors, at each point in space-time we let the basis vector $k^{\alpha}$ be the vector belonging to this congruence. Then $\kappa=\sigma=0$. Here $\Phi$ and $\Psi$ are equal and only (9) and (13) remain non-trivial. Equation (9), when written out in detail, reads

$$
\begin{aligned}
3 \Phi_{2} \Psi_{0} & -10 \Phi_{1} \Psi_{1}+7 \Phi_{0} \Psi_{2}-4 \Delta\left(\kappa \Phi_{1}\right) \\
& +4 \bar{\delta}\left(\sigma \Phi_{1}\right)+\bar{\delta} \delta \Phi_{0}-\Delta D \Phi_{0}+(5 \gamma+\bar{\gamma}-\bar{\mu}) D \Phi_{0} \\
& \quad-(4 \beta+5 \tau) \bar{\delta} \Phi_{0}+(\bar{\beta}-\bar{\gamma}-5 \alpha) \delta \Phi_{0} \\
& +(5 \rho+4 \epsilon) \Delta \Phi_{0}+(4 \Delta \epsilon-4 \bar{\delta} \beta) \Phi_{0} \\
& +(20 \alpha \tau+20 \alpha \beta-20 \rho \gamma-20 \epsilon \gamma-4 \epsilon \bar{\gamma} \\
& \quad+4 \beta \bar{\tau}-4 \beta \bar{\beta}+4 \epsilon \bar{\mu}] \Phi_{0} \\
& +[20 \kappa \gamma+4 \kappa \bar{\gamma}-4 \kappa \bar{\mu}-20 \alpha \sigma-4 \sigma \bar{\tau}+4 \sigma \bar{\beta}] \Phi_{1}=0 .
\end{aligned}
$$

From (4.2b) in [5] it follows that $\Psi_{0}=0$. (14) then reduces to $-10 \Psi_{1}^{2}=0$, showing that $W$ is algebraically special.

Analogue of the Goldberg-Sachs Theorem. To prove the theorem stated in the introduction we have to consider all of the compatibility conditions (9)-(13).

When the Weyl tensor vanishes we can chose the basis null tetrad in such a way that $\kappa=\sigma=0$. We can then investigate the existence of an algebraically general tensor $H$ having the desired properties and satisfying $\Phi_{0}=0$. Since the applicable compatibility conditions (9)-(12) vanish identically, such a tensor certainly exists.

The converse is less trivial to prove. At each point in space-time let us choose the vector of the given congruence as the basis vector $k^{\alpha}$. Then $\kappa=\sigma=0$, and, by the Goldberg-Sachs Theorem, $\Psi_{0}=\Psi_{1}=0$. Given that $H$ is algebraically general, we have to show that $W$ vanishes. We have previously [3] shown that if $\Phi_{0}=0$ and $\Phi_{1} \neq 0$ then $W$ vanishes. This result can be obtained from equations (10)-(12). Let us therefore consider the case $\Phi_{0} \neq 0$. By means of a suitable null rotation about $k^{\alpha}$ we can choose a null tetrad such that $\Psi_{4}$ also vanishes. In this frame $\Psi_{2}$ and $\Psi_{3}$ are the only non-vanishing components of the Weyl tensor.

Equations (10)-(12) now give us two alternatives. Either $\Psi_{2}=\Psi_{3}=0$, in which case the theorem is proved, or $\Psi_{2} \neq 0, \Psi_{3}=\Phi_{1}=\Phi_{3}=0$. In the latter case $\Phi_{4} \neq 0$ since $H$ is algebraically general. By a suitable change of spin-frame as in (5) we can make $\Phi_{0}=\Phi_{4}$. To show that $\Psi_{2}$ cannot be non-zero consider

$$
X_{4}=m^{A} m^{B} m^{C} m^{D} X_{A B C D}
$$


where

$$
X_{A B C D}=5 \Phi_{(A B \mid E F} \Psi^{E F \mid}{ }_{C D)}+S_{A B C D} .
$$

If $X_{4}=0$, equation (9) implies that $\Psi_{2}=0$. If $X_{4} \neq 0$ we perform a null rotation about $k^{\alpha}$ such that $X_{4}^{\prime}=0$. This can be done since $X_{A B C D}$ is an element of $E_{5}$ whose dyad components transform as in (3). In the prime frame the equation corresponding to (13) reduces to

$$
\Phi_{4}^{\prime} \Psi_{2}^{\prime}-\Phi_{2}^{\prime} \Psi_{4}^{\prime}=0 .
$$

From the transformation laws (3) we obtain

$$
\begin{aligned}
& \Psi_{2}^{\prime}=\Psi_{2}, \Psi_{4}^{\prime}=6 c^{2} \Psi_{2}, \quad \Phi_{2}^{\prime}=c^{2} \Phi_{0}+\Phi_{2}, \\
& \Phi_{4}^{\prime}=c^{4} \Phi_{0}+6 c^{2} \Phi_{2}+\Phi_{4},
\end{aligned}
$$

where $c$ is the value of the rotation parameter which makes $X_{4}^{\prime}$ vanish. Substituting in equation (15) we obtain

$$
\Psi_{2}\left[-5 c^{4} \Phi_{0}+\Phi_{4}\right]=0 .
$$

If $\Psi_{2} \neq 0, c^{4}=\frac{1}{5}$ since $\Phi_{0}=\Phi_{4}$. Let us follow the null rotation about $k^{\alpha}$ by one about $m^{\alpha}$ (as in (4)) such that $X_{0}^{\prime \prime}=X_{4}^{\prime \prime}=0$. In this new frame the equation corresponding to (13) is

$$
\begin{aligned}
0 & =\Phi_{4}^{\prime \prime} \Psi_{2}^{\prime \prime}-\Phi_{2}^{\prime \prime} \Psi_{4}^{\prime \prime} \\
& =\frac{24}{5} b c \Psi_{2} \Phi_{0} .
\end{aligned}
$$

If $\Psi_{2} \neq 0, b=0$ and the new frame is identical with the primed frame. From the equation analogous to (9) we then have

$$
\begin{aligned}
0 & =\Phi_{0}^{\prime} \Psi_{2}^{\prime}-\Phi_{2}^{\prime} \Psi_{0}^{\prime} \\
& =\Phi_{0} \Psi_{2} .
\end{aligned}
$$

Thus we are forced to the conclusion that $\Psi_{2}=0$ and the proof of the theorem is complete.

The proof of the corollary immediately follows. A space-time with $W \neq 0$ in which there exists a congruence of geodesic and shearfree null curves allows only the existence of algebraically special tensors $H$ with the desired properties by the theorem just proved. Conversely, if $H$ is algebraically special we can choose the tetrad so that $\Phi_{0}=\Phi_{1}=0$. The Bianchi identities (8) then show that $\kappa=\sigma=0$, i.e. that $k^{\alpha}$ is geodesic and shearfree.

\section{REFERENCES}

[1] M. Chevaluer, Sur l'analogue gravitationnel du théorème de Mariot-Robinson, Comptes Rendus, A263 (1966) 526-528.

[2] G. Ludwia, Généralisation des théorèmes de Mariot, Robinson et Chevalier, Comptes Rendus, A266 (1968) 506-508. 
[3] G. Ludwig, Sur l'analogue gravitationnel du théorème de Mariot-Robinson, Comptes Rendus, A268 (1969) 475-477.

[4] J. N. Goldberg \& R. K. Sachs, A Theorem on Petrov Types, Acta Phys. Polonica, 22 Suppl., (1962) 13-23.

[5] E. Newman \& R. Penrose, An Approach to Gravitational Radiation by a Method of Spin Coefficients, J. Math. Phys., 3 (1962) 566-578.

[6] F. A. E. Pirani, Lectures on General Relativity, Brandeis Summer Institute, 1964, Prentice Hall, 1965.

[7] G. Ludwig, Classification of Electromagnetic and Gravitational Fields, Am. J. Phys., 37 (1969) 1225-1238.

University of Alberta

Edmonton, Canada

Date communicated: ОстовеR 7, 1969 